\title{
Rare Mammary Lesions: A Radiologic-Pathologic Correlation
}

\author{
Ma. Theresa Buenaflor \\ St. Luke's Medical Center, Quezon City
}

\section{ABSTRACT}

Appearances can be deceiving and this pictorial essay illustrates the imaging appearance of breast lesions which may or may not appear as classic for malignancy. These cases are considered unusual, interesting and uncommonly encountered, thus providing an avenue for better collaboration and as a teaching point for both radiologists and pathologists.

Key words: breast neoplasms, angiosarcoma pseudoangiomatous stromal hyperplasia, granulomatous mastitis

\section{INTRODUCTION}

The presentation of unusual breast lesions is quite challenging, knowing that a wide array of benign and malignant lesions may be encountered in practice. Familiarity with their radiologic appearances as well as the pathologic findings are important since the detection of such lesions can impact their clinical management.

Rare breast lesions have mammographic and sonographic features which appear similar to those of breast carcinomas and as such warrant tissue biopsy to obtain a diagnosis.

The four cases included in this report range from benign to malignant lesions that are considered as rare. However, in those that are benign, their imaging features may be similar to those of carcinomas. In light of this, the concomitant pathologic findings augment the imaging, hence, the subsequent diagnosis alters the management.

\section{METHODOLOGY}

This is a case series of four (4) patients with rare breast lesions which have imaging studies that were seen and performed at the Breast Center of St. Luke's Medical Center, Quezon City -one with both digital mammography and ultrasound while the rest only have ultrasound. Their ages range from 24 to 51 years old. The clinical histories were reviewed with particular attention to their initial presentaton as well as their imaging finding. Core-needle biopsies were done and specimens sent to the Institute of Pathology of St. Luke's Medical Center which were then interpreted by a dedicated breast pathologist.

\section{DISCUSSION}

Sarcomas of the breast are extremely uncommon, constituting less than $1 \%$ of all malignant breast tumors and rarely present as primary breast malignancies. The most common breast sarcomas are: phyllodes tumor, previously referred to as cystosarcoma phylloides, and osteogenic sarcoma belong to malignancies of stromal origin. ${ }^{1}$ According to the WHO classification of breast tumors, phyllodes tumors are classified as fibroepithelial tumors, in the same class as fibroadenoma, low-grade periductal stromal sarcoma, and mammary hamartoma while osteosarcoma belongs to tumors classified as mesenchymal tumors which include hemangioma, angiosarcoma, and granular cell tumor to name a few. ${ }^{2}$

Benign phyllodes tumours are characterized by few if any mitoses, moderate to marked cellular overgrowth, and slight to moderate cellular pleomorphism. Low-grade malignant or borderline lesions include a zone of microscopic invasion around their borders, an average of two to five mitoses per 10 high-power field, and moderate stromal cellularity that is heterogeneously distributed in hypocellular areas. Malignant phyllodes tumors show a marked degree of hypercellular stromal overgrowth with more than five mitoses per 10 high-power field, and have an invasive border. ${ }^{3}$ 

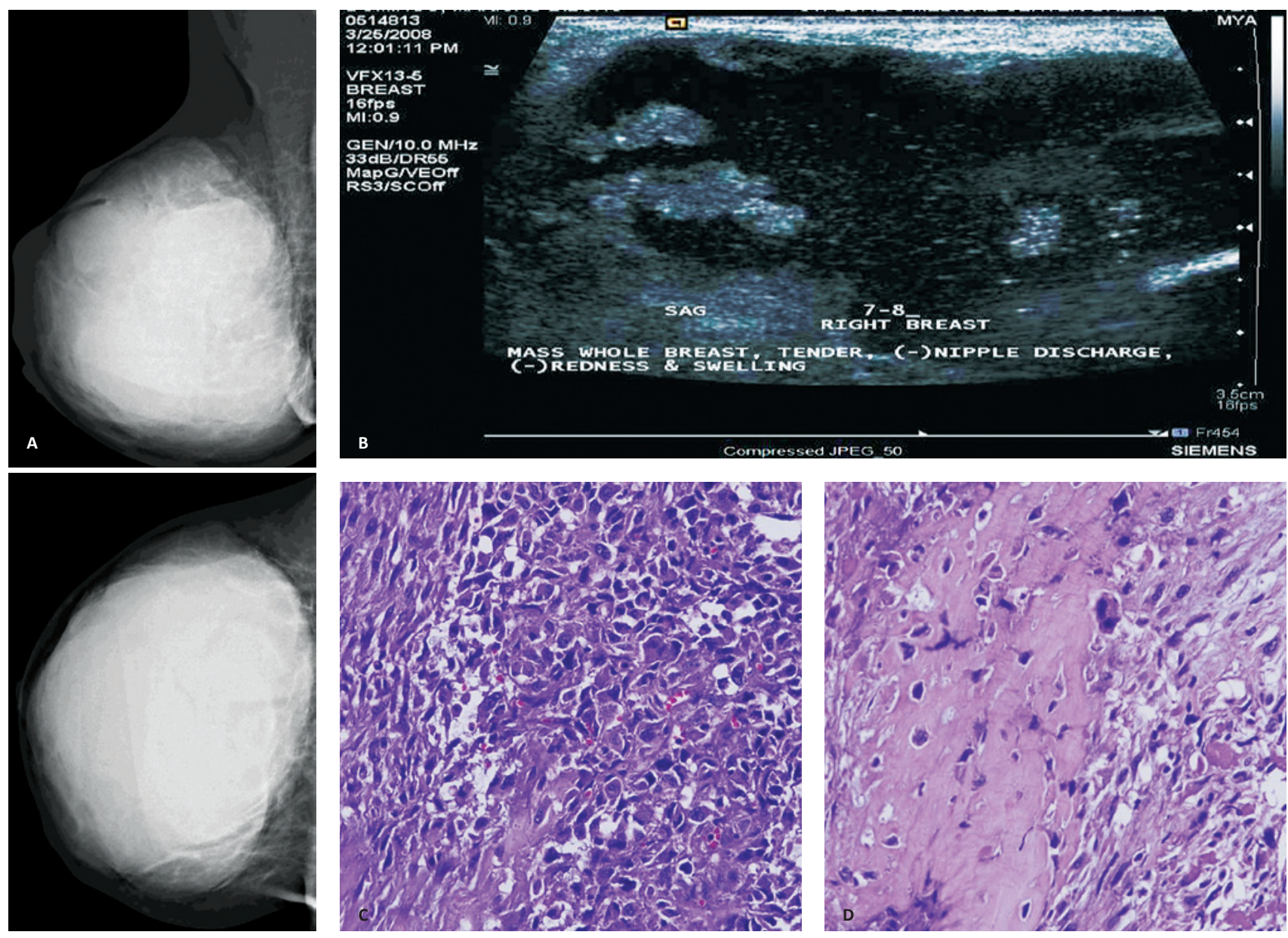

Figure 1. Malignant phyllodes of the breast in a 51-year-old woman with a few month's history of gradually enlarging right breast mass with no history of previous irradiation to the breast/chest region. (A) Mediolateral oblique (MLO) and craniocaudal (CC) mammograms demonstrate the large lobulated mass of high density occupying the entire right breast; (B) Ultrasound (US) image shows a portion of the mass to be complex - with solid and cystic components. The cystic component demonstrates medium to high level echoes; (C) High-power magnification (400x, H\&E) of the cellular component shows it to be made of pleomorphic cells with average mitotic count of 10-12 per hpf; (D) High power magnification of a slide showing a focus with osteosarcomatous features.

Mammographically, phyllodes tumors usually present as focal masses and may have lobulated margins. The tumor may manifest initially as a large mass or may show a rapid increase in size and on ultrasound, a phyllodes tumor may resemble a fibroadenoma or may have a variable appearance with internal heterogeneity, cystic changes, and posterior enhancement. ${ }^{4}$

Primary osteogenic sarcoma can occur in extraosseous locations and has been reported in the breast. They are rare but can occur in an area that was previously irradiated and as many as $40 \%$ of these tumors are preceded by fibroadenomas or cystosarcoma phyllodes. ${ }^{5}$ Osteosarcomatous differentiation in phyllodes tumors is uncommon. ${ }^{6}$ Metastasis occurs by blood rather than by lymphatic spread. Complete excision without axillary dissection is advised.

Pseudoangiomatous stromal hyperplasia (PASH) is a benign lesion that is classified as a mesenchymal tumor of the breast. ${ }^{2}$ The lesion is commonly seen in premenopausal women or those receiving hormone therapy, hence, the lesion is likely related to fluctuations of hormone levels. The clinical manifestation of PASH is myriad, ranging from insignificant incidental microscopic changes in the breast to focal palpable or nonpalpable masslike nodules (nodular PASH) to diffuse breast involvement. ${ }^{4}$ On imaging, US images may show a hypoechoic circumscribed mass that resembles a fibroadenoma. ${ }^{7}$

The most striking histologic finding is a complex pattern of empty anastomosing slitlike spaces within the stroma. These slitlike spaces resemble the vascular spaces in lesions such as low-grade angiosarcoma and may be mistaken for such (hence the name pseudoangiomatous), from which PASH must be histologically differentiated. ${ }^{4,7}$

A short-term follow-up imaging may be done or, alternatively, surgical excision may be performed immediately. The prognosis is generally good, with a reported recurrence rate of approximately $10 \% .^{7}$

Angiosarcoma is a malignancy of endovascular origin. Primary angiosarcoma can arise anywhere in the body. When it occurs in the breast, it affects women in their 3rd and 4th decade and accounts for one in 1700-2300 cases of primary breast cancer. ${ }^{8}$ It arises in the breast more often than in any other organ. ${ }^{3}$

The size and location of palpable masses vary from small cutaneous nodules (the cutaneous subtype) to large lumps that constitute 

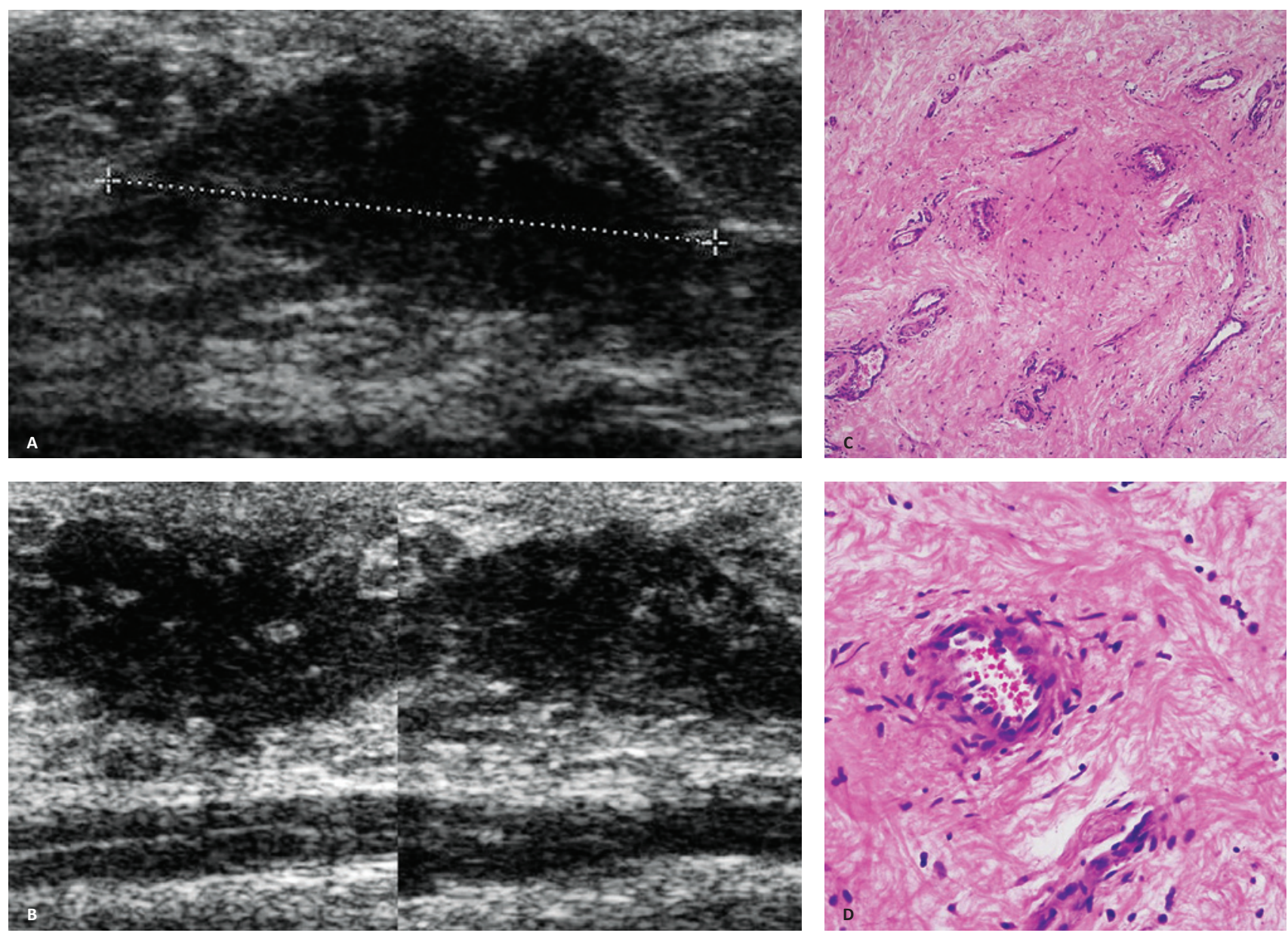

Figure 2. PASH in a 24-year-old woman who presented with palpable masses in both breasts of 3 years duration. (A,B) Ultrasound (US) images demonstrate an irregular hypoechoic solid mass punctuated with hyperechoic foci within the lesion; (C) Low-power magnification (100x, H\&E) shows increased vascularity within the cellular stroma; (D) High-power magnification shows a blood vessel surrounded with typical spindle-shaped cells with normal nuclei (400x, H\&E).

the entire breast (the cutaneous subtype). The majority of cases exhibit skin changes. Advance disease is typically marked by edema and ulcerative lesions in the breast. Radiologic findings are often nonspecific. Mammograms my appear completely normal in $33 \%$. Ultrasound reveals a lesion that may appear hypoechoic, hyperechoic, or a heterogeneous region, with or without acoustic shadowing. ${ }^{8}$

Angiosarcoma is divided into low and high histologic grades. Grade is prognostically siginificant. ${ }^{3}$ The cells of low-grade angiosarcoma resemble endothelium both morphologically and functionally. The cells are typically spindle-shaped and have large, oval nuclei with vesicular chromatin pattern. They form irregular, anastomosing vascular channels, and sometimes seem to connect to dermal capillaries. High-grade angiosarcoma is characterized by larger cells, pleomorphic nuclei, prominent nucleoli, and a high degree of mitotic activity. Hemorrhage into the surrounding stroma, known as "blood lakes," is also common and could account for the sometimes heterogeneous appearance of angiosarcomas on ultrasound. ${ }^{3,8}$

Angiosarcoma although a rare malignancy has a very poor prognosis. It is almost uniformly fatal with rapid metastatic spread and survival beyond 5 years is extremely rare. ${ }^{1}$ Aggressive surgical resection is advocated as the treatment of choice.

Granulomatous mastitis is a very rare inflammatory disease of unknown origin that can clinically mimic carcinoma. It generally manifests as a distinct, firm to hard mass that may involve any part of the breast. The mammographic features are variable, from normal findings to masses with benign or malignant features and focal asymmetric density. The US appearance of multiple clustered, often contiguous tubular hypoechoic lesions is often an uncommon manifestation whose features resemble carcinoma. ${ }^{9}$

At pathologic analysis, granulomatous mastitis manifests as a noncaseating, nonvasculitic granulomatous inflammatory reaction centered on lobules. Granulomatous being the term adopted in the absence of a specific etiologic agent. ${ }^{3}$ Even with the absence of an etiologic agent, it is imperative to take into consideration a pathogenic cause such as tuberculosis which is prevalent in our country. Although the diagnosis of mammary tuberculosis is difficult since acid-fast bacteria are not detected in most cases, it is usually based on inflammatory and granulomatous findings at FNA cytologic analysis or biopsy. Primary treatment consists of excisional biopsy. ${ }^{9}$ 

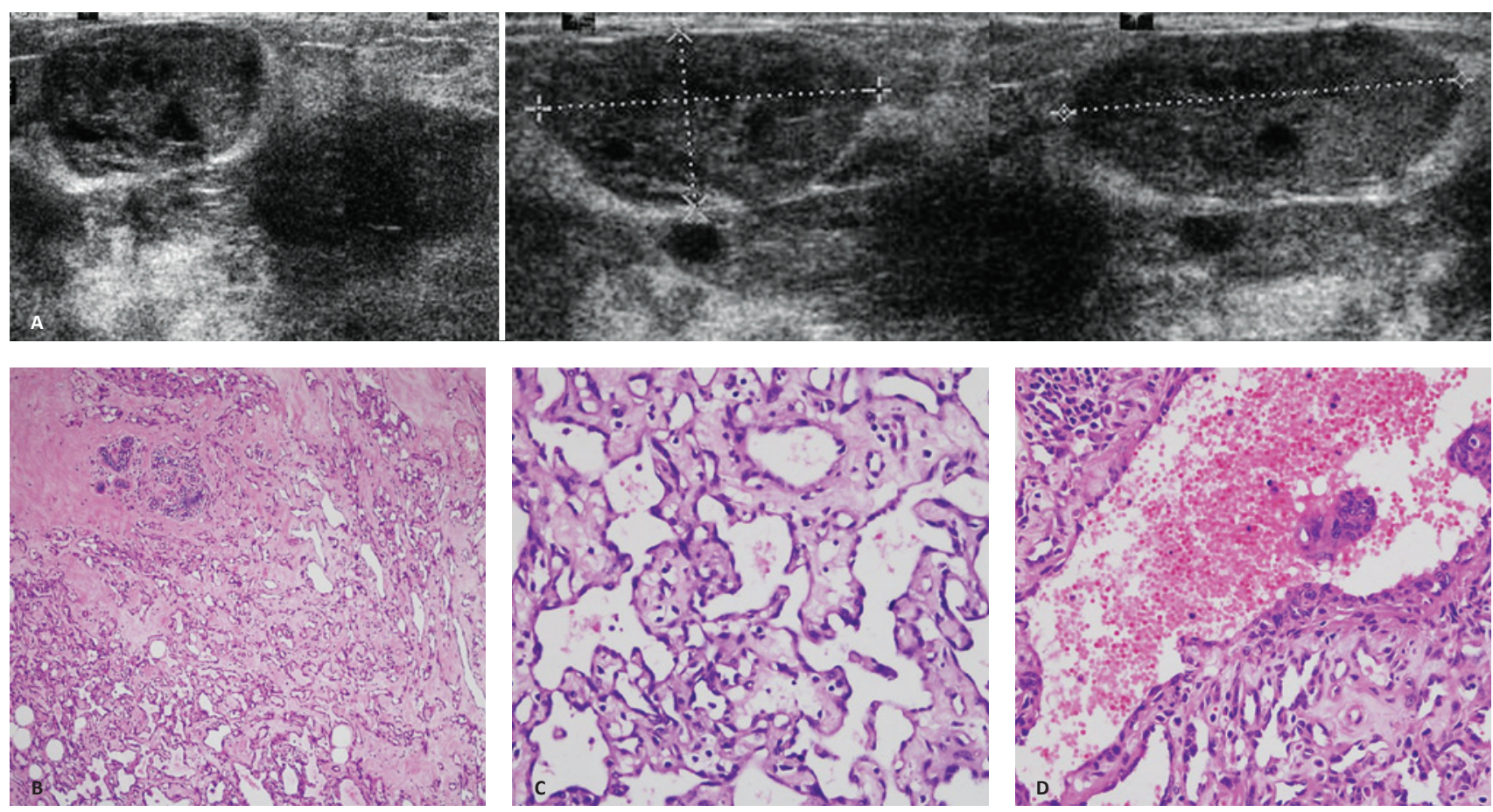

Figure 3. Angiosarcoma of the breast, bilateral in a 32-year-old female who presented initially with a bleeding right breast mass with no history of any previous irradiation to the breast/chest area. (A) US images show fairly circumscribed complex solid nodules with heterogeneous echogenicity, one of which shows few hypoechogenicities within the masses; (B) Low-power magnification (100x, H\&E) shows anastomosing blood vessels; (C) High-power magnification (400x, H\&E) demonstrate atypical spindle-shaped cells lining the vascular channels; (D) HPO view of the typical hemorrhagic "blood lakes" of angiosarcoma (400x, H\&E).
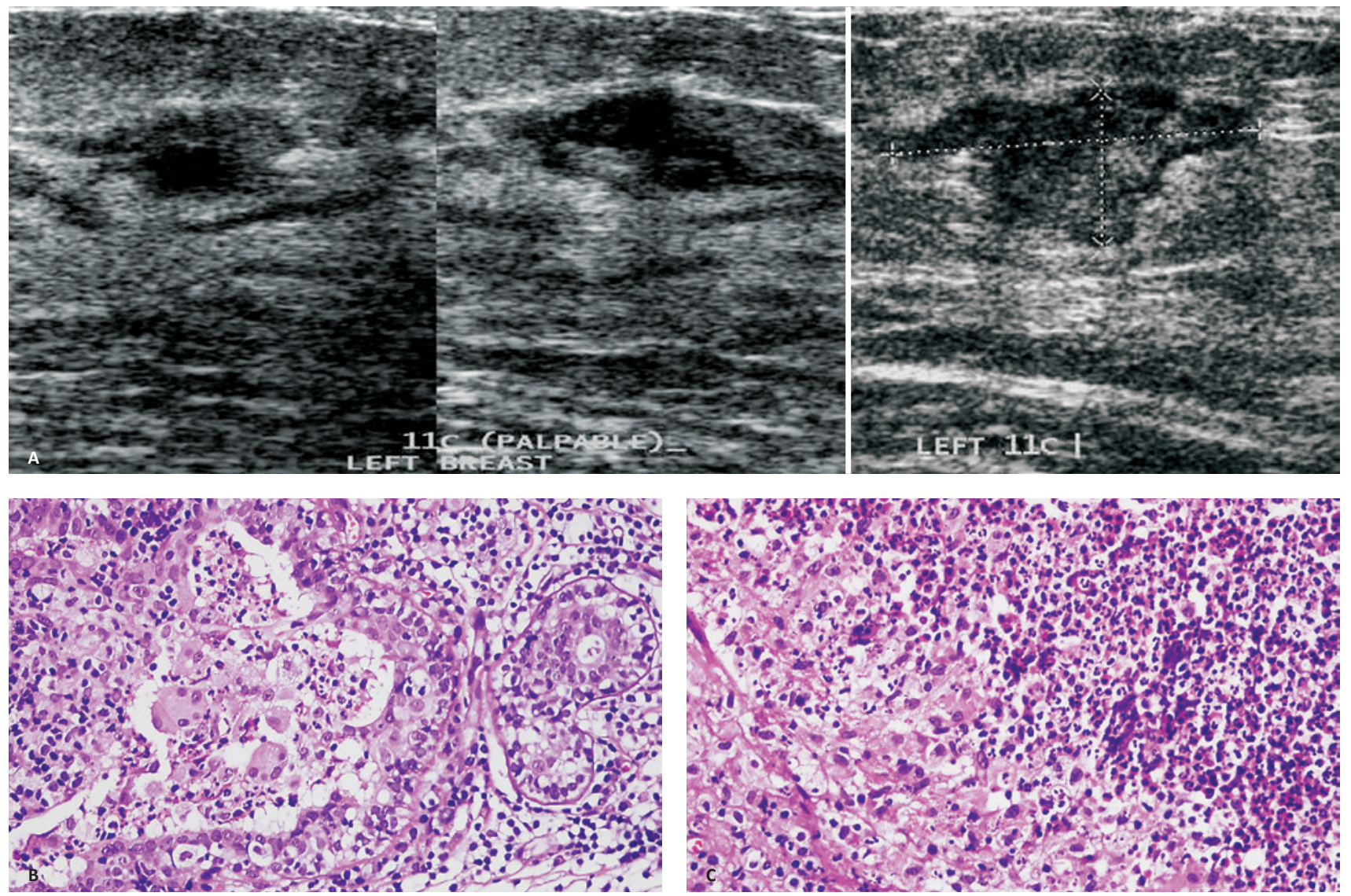

Figure 4. Granulomatous lobular mastitis in a 34-year-old woman who presented with a palpable mass on the left breast. (A) US images demonstrate an irregular hypoechoechogenicity; (B) High-power magnification (400x, H\&E) showing multinucleated giant cells within the terminal ductal-lobular unit; (C) Low-power magnification (100x, H\&E) of an area with proliferation of neutrophils indicating abscess with disruption of the basement membrane. 


\section{CONCLUSION}

Radiologic-pathologic correlation is crucial in the diagnosis of the spectrum of breast diseases. The relation of the underlying pathology of a lesion explains its imaging appearance. However, as these cases have demonstrated, lesions that initially present as malignancy may actually be benign or vice versa. Close collaboration between radiologists and pathologists is copacetic and greatly influences clinical decisions and management.

\section{AUTHOR DISCLOSURE}

The author wrote this case report while she was a fellow of breast imaging at the Breast Center of St. Luke's Medical CenterQuezon City. There are no other conflicts of interest declared.

\section{ACKNOWLEDGMENT}

The author thanks the breast imaging specialists of the Breast Center at St. Luke's Medical Center-Quezon City: Drs. Mercy GoSanti and Barbara Helen Escano-Perez. The breast surgeons who graciously shared their cases: Dr. Roel Tolentino, Dr. Genevieve Padilla-Evangelista, and Dr. Michelle Uy and the pathologists Dr. Manuelito A. Madrid (breast pathologist) and Dr. Jasper Andal who kindly provided the images from slides and elucidated the pathologic interpretation.

\section{REFERENCES}

1. Kopans D. Breast imaging 3rd ed. Philadelphia, PA: Lipincott Williams \& Wilkins. 2007.

2. Tavassoli FA, Devilee P, eds. Tumours of the breast. In: Pathology and genetics of tumours of the breast and female genital organs. WHO classification of tumours. Lyon, France: IARC; 2003;9-112.

3. Rosen PP. Rosen's breast pathology 2nd ed. Philadelphia, PA: Lipincott Williams \& Wilkins, 2001.

4. Irshad A, Ackerman SJ, Pope TL, et al. Rare breast lesions: correlation of imaging and histologic features with WHO classification. Radiographics. 2008;28(5):1399-1414. http://dx.doi.org/10.1148/rg.285075743.

5. Watt AC, Haggar AM, Krasicky GA. Extraosseous osteogenic sarcoma of the breast: mammographic and pathologic findings. Radiology. 1984;150(1):34. http://dx.doi. org/10.1148/radiology.150.1.6580676.

6. Silver SA, Tavassoli FA. Osteosarcomatous differentiation in phyllodes tumors. Am J Surg Pathol.1999;23(7):815-21. PMID: 10403305.

7. Goel NB. Knight TE, Pandey S. Riddick-Young M, et al. A. fibrous lesions of the breast: imaging-pathologic correlation. Radiographics. 2005;25(6):1547-59. http://dx.doi.org/10.1148/ rg.256045183.

8. Lim RF, Goei R. Angiosarcoma of the breast. Radiographics. 2007;27(1):S125-30. http://dx.doi.org/10.1148/rg.27si075016.

9. Sabate JM, Clotet M. Gómez A, et al. Radiologic evaluation of uncommon inflammatory and reactive breast disorders. Radiographics. 2005;25(2):411-24. http://dx.doi.org/10.1148/ rg. 252045077 .

Disclaimer: This journal is OPEN ACCESS, providing immediate access to its content on the principle that making research freely available to the public supports a greater global exchange of knowledge. As a requirement for submission to the PJP, all authors have accomplished an AUTHOR FORM, which declares that the ICMJE criteria for authorship have been met by each author listed, that the article represents original material, has not been published, accepted for publication in other journals, or concurrently submitted to other journals, and that all funding and conflicts of interest have been declared. Consent forms have been secured for the publication of information about patients or cases; otherwise, authors have declared that all means have been exhausted for securing consent. 